\title{
Age differences in the acquisition and retention of concurrent discriminations by monkeys
}

\author{
LEE BAKNER and F. ROBERT TREICHLER \\ Kent State University, Kent, Ohio
}

\begin{abstract}
The performances of adult and aged macaque monkeys were compared on several tasks requiring acquisition and retention of concurrent object discriminations. Cross-sectional and longitudinal comparisons between 11-year-old and over-20-year-old animals were conducted on the acquisition of several 16-problem concurrent tasks, and after 2 weeks, retention by the different age groups was also evaluated. Although the retention tests indicated no age-related impairment, acquisition tests showed that aged animals made reliably more errors-to-criterion than did adult animals. The relative contributions of intersession and intrasession improvement and preference phenomena were considered and contrasted to some previously reported age-specific outcomes. Although the present results affirmed the contention that old animals were selectively impaired on intersession task requirements, some earlier interpretations of preference effects were questioned. Old animals did not show a potentiation of specific object preferences; rather, they tended to be nonsystematic in maintaining their selection of initially preferred objects. Concurrent discrimination was indicated to be a behavioral measure that is, under appropriate circumstances, selectively sensitive to aging and may offer an appropriate comparative model of informationprocessing characteristics.
\end{abstract}

Decline in performance on learning and memory tasks has been a persistent concern when investigating the issue of aging (e.g., Kubanis \& Zornetzer, 1981; Stein, 1980 ), and although questions have been raised regarding the comparability of animal outcomes (see Bartus, 1988 , for a review and clarification of this issue), nonhuman primate behavior has sometimes been proffered as a model of the human aging process (Bartus, 1979; Davis, 1978). Age-related performance decrements in primates have been noted in a variety of situations including iconic memory tests (backward masking; Davis, 1974, p. 167-169), short-term memory performances (delayedresponse tasks; King \& Michels, 1989; Medin, 1969), and long-term retention of discrimination tasks (Medin, O'Neil, Smeltz, \& Davis, 1973).

A contrast of outcomes on some long-term retention tests reveals the kind of difficulty sometimes encountered in interpreting age-related results. Medin et al. (1973) and Davis (1974, pp. 170-173) tested precisely the same animals on 24-h retentions and reached different conclusions. Davis trained animals to criterion on a pattern-generation task that required monkeys to select elements in a display matrix, and he then evaluated retention by requiring animals to regenerate the pattern on the following day.

Portions of this work served as the first author's master's thesis at Kent State University and were presented as a paper at the Midwestern Psychological Association meetings in 1989. The authors wish to acknowledge the assistance of $Y$. Hines in animal care and testing. We also express appreciation for the very constructive suggestions of our anonymous Reviewer A. Reprint requests may be addressed to either author at the Department of Psychology, Kent State University, Kent, $\mathrm{OH} 44242$.
Both adult and aged monkeys performed with equal facility, and it was concluded that advanced age did not impair 24-h retention. Medin et al. required the monkeys to learn at least 15 concurrently presented problems with 3 or 4 appearances of each problem in a daily session. Performances on a series of four tasks were equivalent, but adult and aged groups differed on their day-to-day improvement within each task. Although they did not differ on first-day performances, the old animals committed more errors than did adults on each of 3 subsequent test days. It was suggested that old monkeys were impaired because of their more rapid forgetting during the 24- $\mathrm{h}$ intersession intervals.

In an attempt to comprehend the nature of the intersession losses of these old animals, the conditional probabilities of correct choice after right or wrong selections on the immediately preceding trial were determined for each problem. The investigators compared measures from the last two intrasession trials to measures of transition from the last trial of one session to the first trial of the next session. Old and middle-aged animals differed only on the intersession (not intrasession) transitions, and only after an incorrect choice. That is, old animals showed the distinctive characteristic of greater error on those problems that had been wrong on the last trial of the previous day. Accordingly, old animals' day-to-day losses were attributed to their forgetting the previous day's reward contingencies and their tendency to continue making choices on the basis of object preferences as indicated by incorrect choice on the last trial of the previous session. Unfortunately, the measure upon which this conclusion was based has some difficulties. One problem was that the occurrences of intertrial transitions (based on last-trial 
error) were of a small and indeterminate number, and even these should have reduced as training proceeded.

It should also be noted that the Davis (1974, pp. 170173 ) and Medin et al. (1973) studies measured retention at different times during the course of training. Davis required attainment of an acquisition criterion before evaluating the retention shown by adult and aged monkeys, whereas Medin et al. compared measures of improvement over 4 days of training on each task without employing acquisition criteria. Perhaps the age-differentiated results of Medin et al. were specific to acquisition performances and would not be evident in a test administered following attainment of proficient initial performance.

Within the present investigation, it was our intent to focus upon some of the issues noted in these previous successes and failures in detecting age-specific outcomes. Accordingly, we chose to measure performance on a multiple-stimulus task, in part because review of the literature suggested that reliable detection of age-differentiated outcomes was most likely to appear when multiple stimuli were used, not only in tests of long-term memory, but also when iconic and short-term memory tests had been employed (Bartus \& Dean, 1979; Davis, 1974, pp. 167169; Medin, 1969; Moss, Rosene, \& Peters, 1988). The concurrent discrimination task seemed an appropriate choice because it entailed presentation of multiple stimuli and also allowed specification of acquisition and retention phases of training. This was accomplished by arranging tasks so that an acquisition criterion could be imposed and the retention test could be temporally separated from the training phase. Thus, we hoped to clarify some of the issues regarding the interactive contribution of age and training phase that were raised in the contrast of the Davis (1974, p. 170-173) and Medin et al. (1973) results.

Additionally, the concurrent paradigm entails features that allow manipulation of object-preference characteristics, and we attempted to evaluate their contribution to the various phases of discrimination performance. In the present study, reluctance to abandon preferences that existed at the initiation of training was assessed by imposing the baited/unbaited first-trial procedure of Bessemer and Stollnitz (1971). This required that on the first appearance of the set of problems, rewards were under both of the objects for a randomly selected half of the problems (baited), while no rewards were beneath either object for the other half of the problems (unbaited). Choices on the first trial of each problem then determined subsequent correct alternatives. Baited (rewarded) choices were correct on all their subsequent appearances, while the nonchosen objects of unbaited first trials covered rewards on their later appearances. Initial choices thus defined sets of problems requiring choice of initially preferred or nonpreferred objects for subsequent solution, and relative difficulty of the different problem types could be compared. If aged animals were especially reluctant to abandon initially preferred object selections, then they might be expected to commit relatively more errors on the unbaited problem type.
The present study also took advantage of our laboratory's history of concern for evaluating primates' capacities to learn and retain problems presented via the concurrent procedure (Treichler, 1984; Treichler, Wetsel, \& Lesner, 1977). Although all monkeys had extensive experience on concurrent discrimination, an "old" group of monkeys in this study had been trained on comparable concurrent tasks approximately 9 years prior to the present measures of concurrent performance. This allowed longitudinal comparisons of performance between the same subjects trained earlier as adults and later when aged. Additionally, another younger, but sophisticated, group of adult monkeys was contemporarily trained and tested on the same problems as the aged subjects and provided cross-sectional comparisons between the two independent groups of subjects.

\section{METHOD}

All testing was conducted in a manual Wisconsin General Test Apparatus (WGTA) using pairs of multidimensionally different, common-use objects like those of Treichler et al. (1977). These discriminanda were placed in channels on the form-board tray in a manner that required the monkey to grasp and displace an object in order to indicate a choice response. Correct choices were rewarded by any one of a variety of incentives, including raisin, apple, or sugared cereal segments. All tasks were composed of 16 different pairs of objects that appeared as simultaneous, two-choice problems, and trials were terminated immediately after an animal's initial choice response (i.e., noncorrectional testing). Object pairs were maintained throughout a task, but object and reward locations were counterbalanced within the set of 16 problems.

Two separate groups of rhesus monkeys (Macaca mulatta) with extensive prior testing experience on concurrent object discrimination tasks served as subjects. An old group was composed of 4 female monkeys originally obtained from commercial suppliers in 1973. These subjects were estimated to be between 20 and 23 years old, or perhaps even older, at the time of the present (1988) tests. Five other female monkeys, all born in 1977, were added to the colony in 1980 and formed the adult (11-year-old) group of the present study. For each animal, designation of the correct object within a problem was accomplished by using the baited/unbaited first-trial procedure of Bessemer and Stollnitz (1971). Accordingly, on each problem's first appearance, 8 of the 16 problems were presented with food under both objects (the baited problem type), and the other 8 problems were presented with no food under either object (the unbaited problem type). Thus, for all baited problems, initially selected objects were designated as correct, whereas for all unbaited problems, initially nonchosen objects were correct on their subsequent appearances. This systematic designation of correct alternatives allowed evaluation of object preference as an influence upon subsequent acquisition and retention performance.

On the day following first-trial preference determination, concurrent training was initiated. During an animal's daily session, each object pair appeared twice (yielding 32 trials per session), and the entire 16-problem set was presented before it reappeared in a different nonsystematic order. This phase of training continued until a criterion of errorless performance on one run through the 16problem task was achieved or until 8 days of training were completed. Retention was evaluated by a relearning test administered 10-14 days after acquisition and entailed reattainment of the original criterion.

Those monkeys in the group designated as old had acquired two similar 16-problem tasks when they were 11-14 years old in 1979. 
Then, in 1988, these same subjects, now 20-23 years of age, were trained on each of two 16-problem tasks (analogous to the tasks provided in 1979) and were tested 10-14 days later for retention of these tasks. The 11-year-old adult group's acquisition and retention performance was evaluated on the same two 16-problem tasks presented to the old group in 1988. Thus, it was possible to make comparisons between the old group's acquisition performances when adult in 1979 and their acquisition performances on similar concurrent tasks as aged subjects in 1988 (a longitudinal comparison). Further, cross-sectional comparisons of performance were possible between the two independent groups. Concurrent acquisition by the old group (when adult) in 1979 could be compared to the adult group's performance in 1988 . Additionally, concurrent acquisition and retention by the old group (when aged) could be compared to the adult group's performance by using results obtained in the sequence of training and testing conducted in 1988.

\section{RESULTS}

\section{Acquisition}

Errors to criterion served as the primary dependent measure in the initial statistical analyses. To detect possible age-related differences in acquiring the two 16problem tasks, the error scores for the independent groups of subjects (adult ' 88 vs. old '79; adult ' 88 vs. old ' 88 ) were examined using mixed $2 \times 2$ analyses of variance (ANOVAs) employing age and task as factors. These cross-sectional comparisons revealed that when both groups were adult (adult ' 88 vs. old '79), they showed no significant differences in performance $[F(1,7)=.22$, $p>.50]$. However, in 1988, the old animals committed more errors to criterion than did adult subjects when both groups were trained on the same concurrent tasks $[F(1,7)=8.2, p=.024]$. Similarly, a repeated measures ANOVA for the longitudinal comparison in the present study indicated that the subjects trained when adult and later when aged (old '79 vs. old ' 88 ) made more acquisition errors when old in contrast to their own performance
9 years earlier $[F(1,3)=13.9, p=.036]$. Because two concurrent tasks were always presented in our training sequences, it was possible to compare differences between the first and second task acquisitions. These ANOVAs revealed that performances on the two tasks never differed significantly ( $p s>.05$ ), and there were no statistically significant interactions between the age and task order variables $(p s>.05$ ). Accordingly, a mean errorsto-criterion score on the two 16-problem tasks was computed for each animal and used in all subsequent analyses. Table 1 displays both individual and group mean acquisition errors-to-criterion scores for the various groups and shows that the old group trained in 1988 committed more acquisition errors compared to their own performances in 1979 and to a separate group of adult monkeys in 1988 .

Further analyses attempted to determine the source of the age differences in acquiring concurrent tasks. Courses of acquisition over the $\mathbf{8}$ days of training were examined for the various age groups (see Figure 1) using appropriate ANOVA procedures.

These analyses confirmed the previously reported age effects and revealed improvement in all groups' performances across days of acquisition, producing a main effect of days of acquisition for adult ' 88 versus old ' 79 $[F(7,49)=57.0, p<.001]$, for adult ' 88 versus old ' 88 $[F(7,49)=72.1, p<.001]$, and for old ' 79 versus old '88 $[F(7,21)=28.0, p<.001]$.

When the interaction of age and days of training was evaluated, it was found that aged animals showed less improvement across training days relative to adult subjects. This interaction approached statistical significance for the comparison between adult and old animals tested in 1988 $[F(7,49)=1.9, p=.096]$ and was statistically significant for the comparison between the old group's performance in 1979 versus their own performance in 1988 $[F(7,21)=3.3, p=.017]$. Contrastingly, no such inter-

Table 1

Mean Baited/Unbaited and Total Error Scores in Acquisition and Retention of 16-Problem Tasks for Monkeys in the Various Groups

\begin{tabular}{|c|c|c|c|c|c|c|c|}
\hline \multirow[b]{2}{*}{ Group } & \multirow[b]{2}{*}{ Subject } & \multicolumn{3}{|c|}{ Acquisition } & \multicolumn{3}{|c|}{ Retention } \\
\hline & & Baited & Unbaited & Total & Baited & Unbaited & Total \\
\hline \multirow[t]{2}{*}{$\begin{array}{l}\text { Old monkeys '79 } \\
\text { (when adult) }\end{array}$} & $\begin{array}{l}\text { A } \\
\text { B } \\
\text { C } \\
\text { D }\end{array}$ & $\begin{array}{r}10 \\
8 \\
8 \\
13\end{array}$ & $\begin{array}{l}35 \\
39 \\
19 \\
26\end{array}$ & $\begin{array}{l}45 \\
47 \\
27 \\
39\end{array}$ & & & \\
\hline & Overall mean & 10 & 30 & 40 & & & \\
\hline \multirow[t]{2}{*}{$\begin{array}{l}\text { Old monkeys ' } 88 \\
\text { (when aged) }\end{array}$} & $\begin{array}{l}\text { A } \\
\text { B } \\
\text { C } \\
\text { D }\end{array}$ & $\begin{array}{l}26 \\
22 \\
38 \\
16\end{array}$ & $\begin{array}{l}52 \\
50 \\
49 \\
42\end{array}$ & $\begin{array}{l}78 \\
72 \\
87 \\
58\end{array}$ & $\begin{array}{r}9 \\
1 \\
14 \\
5\end{array}$ & $\begin{array}{r}7 \\
10 \\
17 \\
16\end{array}$ & $\begin{array}{l}16 \\
11 \\
31 \\
21\end{array}$ \\
\hline & Overall mean & 26 & 48 & 74 & 7 & 13 & 20 \\
\hline \multirow[t]{2}{*}{ Adult monkeys ' 88} & $\begin{array}{c}\mathbf{E} \\
\mathbf{F} \\
\mathbf{G} \\
\mathbf{H} \\
\mathbf{I}\end{array}$ & $\begin{array}{r}4 \\
7 \\
8 \\
23 \\
16\end{array}$ & $\begin{array}{l}21 \\
34 \\
22 \\
39 \\
46\end{array}$ & $\begin{array}{l}25 \\
41 \\
30 \\
62 \\
62\end{array}$ & $\begin{array}{l}1 \\
0 \\
7 \\
7 \\
3\end{array}$ & $\begin{array}{r}8 \\
11 \\
17 \\
25 \\
11\end{array}$ & $\begin{array}{r}9 \\
11 \\
24 \\
32 \\
14\end{array}$ \\
\hline & Overall mean & 12 & 32 & 44 & 4 & 14 & 18 \\
\hline
\end{tabular}




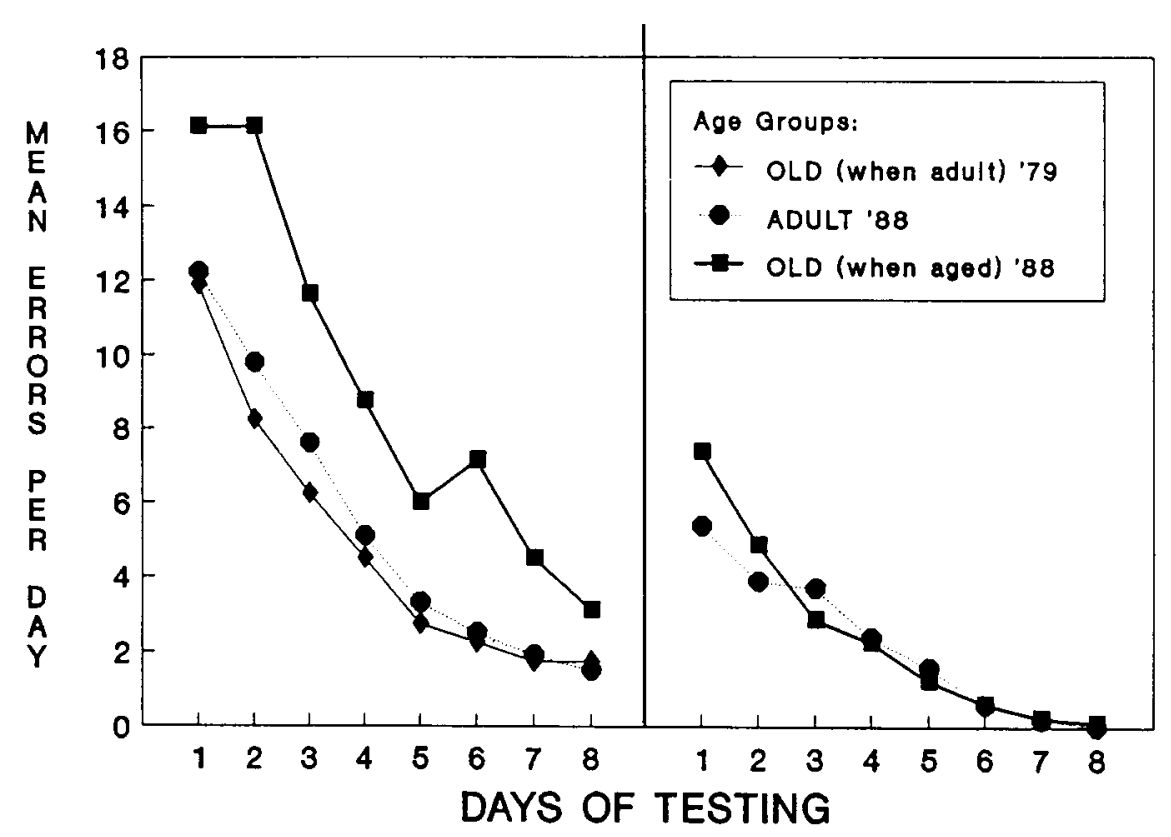

Figure 1. Mean errors per day during acquisition and retention of 16-problem tasks by the various age groups.

action was obtained when the two groups of monkeys were of similar age, adult ' 88 versus old ' $79[F(7,49)=.36$, $p>.50]$.

Post hoc tests were conducted to identify those acquisition days where age differences existed. Using NewmanKeuls tests, significant differences were obtained between the adult and old groups' acquisition scores in 1988 on Training Days $1,2,3,4$, and 6 ( $p s<.05$ ). Similar Newman-Keuls tests also revealed that the old group in 1988 committed significantly more errors than they had when adult in 1979 on Acquisition Days 1-7 ( $p$ s < .05). Apparently, aged animals acquired concurrent tasks at a slower rate than adults; however, if given the opportunity (i.e., 8 days of training), aged animals attained levels of performance equivalent to those of adults.

The present training measures also allowed evaluation of age influences on object preferences, and Table 1 displays the individual and group mean scores on initially rewarded (baited) and nonrewarded (unbaited) problems in acquisition. When examining these scores, the small amount of error entailed in acquiring the baited problems relative to the larger amount of error required to learn the unbaited problems was immediately evident. Significantly fewer errors were associated with baited as compared with unbaited problems for all possible comparisons, producing a main effect of baited/unbaited problem type for adult ' 88 versus old '79 $[F(1,7)=48.6$, $p<.001]$, for adult ' 88 versus old ' $88[F(1,7)=68.1$, $p<.001]$, and for old ' 79 versus old ' $88[F(1,3)=$ $27.4, p=.017]$. These findings are consistent with Bes- semer and Stollnitz's (1971) contention that initial object preferences influence acquisition of concurrent problems.

Because it has been suggested that one factor contributing to the performance impairments of aged animals might be their reluctance to abandon choice of initially preferred objects (Medin et al., 1973), evaluating the interaction of the age and problem-type variables was of particular concern. If one speculated that aged animals perseverate by repeatedly selecting initially preferred, but incorrect, objects, then the baited/unbaited first-trial procedure might reveal a preferential response factor if aged, in contrast to adult, monkeys were to commit relatively more errors on the unbaited problems. However, for all of the comparisons between the age groups, no interactive effect of age and problem-type variables was observed on any of the tasks, that is, there was a nonsignificant age $\times$ baited/unbaited problem-type interaction for adult ' 88 versus old ' $79[F(1,7)=.02, p>.50]$, for adult ' 88 versus old ' $88[F(1,7)=.28, p>.50]$, and for old '79 versus old ' $88[F(1,3)=1.0, p=.388]$, and the age impairment reported here did not seem to depend upon simple perseverative selection of initially chosen objects.

Other properties of the task-presentation format have allowed us to analyze several prospective bases for the different performances shown by adult and old animals while attaining acquisition criteria. For example, because a concurrently acquired set of 16 problems was presented twice within a daily session, a question could be posed as to the differences in the amounts of within-session improvement shown by adult and aged animals. Similarly, 


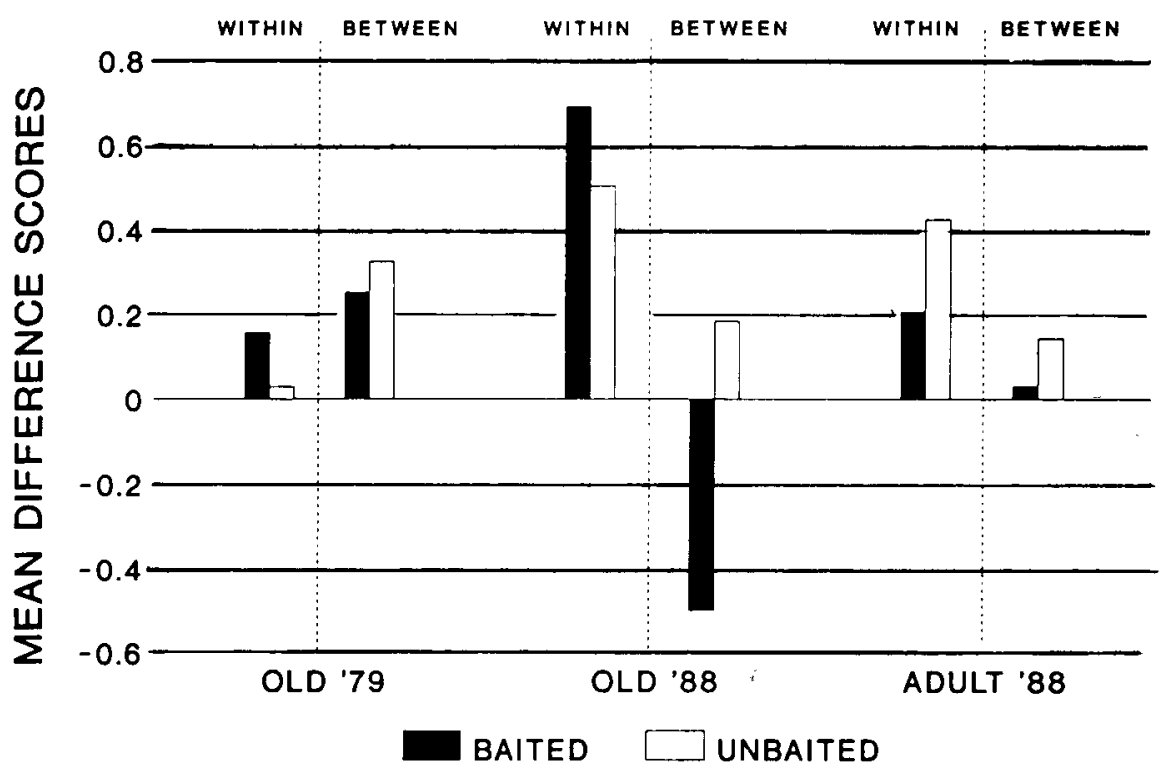

Figure 2. Mean error difference between successive presentations for baited and unbaited problems on between- and within-session transitions by the various age groups during acquisition.

the prospect of differential error reduction between the trials that appeared as the last presentation of one day and the first presentation of the next day could be evaluated. Furthermore, because the baited/unbaited initial-exposure procedure allowed specification of half of the problems within each task as ones learned with or against initialobject preferences, these characteristics could be evaluated in the same analysis as the within-session and between-session error transitions. As an index of transition in error during acquisition, we took difference measures between the successive presentations of each problem during the course of learning 16-problem tasks. For each subject, we were able to generate a score that represented the change in number of errors on the 8 baited or unbaited problems in a task over the 8 days of original training. It was also possible to categorize these scores as withinsession transitions (mean error difference between problems seen on first and second presentations within the same day averaged across the 8 days) or between-session transitions (mean error differences between problems seen as the second presentation of one day and the first presentation of the next day averaged across the seven dayto-day transitions). Although there was a slight tendency toward greater change on the earlier days of training, the overall similarity of difference scores on the several days of acquisition seemed to obviate statistical analyses of trends across the course of training. Rather, our averaged difference scores were used to conduct $2 \times 2 \times 2$ ANOVAs that compared various age groups (adult vs. aged) when the different types of transitions (within vs. between sessions) were imposed for both problem types (baited vs. unbaited). Figure 2 displays mean transition difference scores for each of the groups, and, as was done in treat- ing errors-to-criterion measures, a separate ANOVA for each intergroup comparison was conducted.

Both of the comparisons involving contrasts of aged and adult outcomes revealed similar patterns of differences. Whenever old and adult animals were compared, significant main effects of the baited/unbaited and the between-/within-session conditions were obtained, and the difference measures did not yield a main effect of age. However, in both of these analyses, age did influence performance, as indicated by a significant interaction of age and transition type (within vs. between session) influences. Figure 2 reveals that the old ' 88 group was very different from younger groups in the way they reduced their errors on the within- and between-session transitions. The old group showed distinctive performances on the baited problems between sessions, as suggested by a marginally significant three-way interaction $[F(1,7)=5.2, p=$ .054 ] in the analyses of adult and old subjects in 1988 . Old animals did not consistently reduce error between sessions; rather, they showed an increase in error between sessions, but only on the baited problems. Of course, because these animals eventually learned and performed as well as adult groups by the last day of training, they needed to show improvement, especially on within-session, baited problems. Indeed, they seemed to do this, and when the old ' 88 and adult ' 88 groups were compared, NewmanKeuls tests showed that they differed reliably on baited problems both within and between sessions. Comparisons between the old ' 88 group and their own performances as old ' 79 revealed generally similar outcomes on the transition measures, although their greater variability did not yield the same pattern of statistical significances seen on the 1988 intergroup comparisons. 
The two groups tested as adults were also compared (adult ' 88 vs. old ' 79 ) in a $2 \times 2 \times 2$ ANOVA and showed no significantly different main effects. There was a significant group $\times$ transition type interaction based on the somewhat greater within-session versus between-session improvement of the adult ' 88 group and the opposite pattern in the old ' 79 group.

\section{Retention}

The outcomes of retention tests for adult and old groups tested in 1988 are also included in Table 1. These scores suggest little difference in retention between aged and adult animals tested on the same 16-problem tasks. A mixed ANOVA of adult and old groups' retention performances in 1988 confirmed that there were no agerelated differences in retention $[F(1,7)=.09, p>.50]$, a significant effect of days of retention testing $[F(7,49)=$ $42.7, p<.001]$, and no significant age $\times$ days of testing interaction $[F(7,49)=1.6, p=.154]$. Furthermore, a repeated measures ANOVA between acquisition and retention scores for both groups indicated significantly fewer overall errors when the subjects were reacquiring the tasks $[F(1,8)=34.1, p<.001]$ and suggested substantial savings.

Table 1 also displays the individual and group mean baited/unbaited scores in retention for adult and old groups tested in 1988. These scores did show that significantly fewer errors were committed on the baited as compared with unbaited problems in reacquiring concurrent tasks. A mixed ANOVA of age and problem type indicated that although there were no age-related differences in retention $[F(1,7)=.09, p>.50]$, there was a significant effect of problem type, with fewer errors committed on baited problems $[F(1,7)=19.0, p=.004]$, but there was no significant age $\times$ problem type interaction $[F(1,7)=$ $2.8, p=.157$ ).

One further analysis compared performances of the old and adult groups in 1988 on the last acquisition day versus the first retention day for both the baited and unbaited problem types. This $2 \times 2 \times 2$ ANOVA revealed a significant difference between scores on the acquisition and retention tests, with greater error observed on the first retention day $[F(1,7)=36.8, p<.001]$. There was also a significant main effect of problem type, with greater error shown on unbaited problems $[F(1,7)=18.4, p=$ $.004]$. However, once again, there was no significant difference between the two age groups $[F(1,7)=1.8, p=$ $.22]$, nor were there any significant interactions.

\section{DISCUSSION}

In the present tests, both cross-sectional and longitudinal measures revealed age-related deficits in the acquisition of 16-problem concurrent tasks. However, if aged monkeys received sufficient amounts of training, they showed no impairment relative to adults on a retention test administered 2 weeks later.
The general properties of acquisition displayed by both younger and older animals were similar to those seen in previous tests of concurrent discrimination. The now-welldocumented pattern of greater error on problems requiring choice of an object different from the one selected on first exposure (i.e., unbaited problem type) was evident for all animals in all tests. Although older animals committed more errors on the earlier days of training, they achieved proficiency equivalent to that of adults after 8 days of training. Furthermore, the overall levels of error committed by old animals did not depend upon a selective deficit associated with those problems designated as unbaited, and, therefore, advanced age was not accompanied by a potentiation of initial object preferences. However, the analysis of changes that took place over the successive problem presentations during the course of acquisition did offer a prospective attribution for the observed age-related deficit. Old monkeys seemed to reduce error as much as (or more than) adult animals on the repeated presentation of a list of problems within a daily session. In contrast, and in keeping with the suggestion of Medin et al. (1973), old animals were less proficient than adults when the task required them to retain information from one session to the next. However, performance impairment over a 24 -h intersession interval was not evident on all the problems they learned; rather, errors were more likely to occur on those problems where the initial choices had been rewarded (i.e., the baited problem type). On problems that required choice of an initially nonpreferred object (i.e., unbaited problem type), old animals were no different from adults in reducing error either within or between days. This pattern of problemspecific error is in some contrast to Medin et al.'s results regarding old animals' failure to improve from day to day. They attributed errors to a persistent reluctance to select nonpreferred objects, but the present results found that the old animal's predominant error was one of failing to maintain choice of initially preferred objects. Although sensitive to nonreward and able to choose correctly on the second trial of a session (i.e., they showed substantial within-session improvement on baited problems), old animals seemed relatively poor at retaining and utilizing information about baited problems on the next day.

It also seems appropriate to consider the possibility that nonassociative performance factors might have contributed to the acquisition impairment of our aged animals. Among factors suggested to operate in this fashion are decreased motivation, sensory and/or motor impairments, and attention deficits. Although certainly worthy of consideration, most investigations have reported that performance factors are not typically responsible for agerelated learning and memory impairments. For example, those manipulations designed to evaluate level of motivation, such as physical measures of effort to obtain reinforcers or amount of food deprivation, have yielded nondifferential performances in old and adult monkeys (Bartus, Fleming, \& Johnson, 1978; Medin, 1969). Bartus 
et al. (1978) also reported that aged animals exhibited sensory-detection properties very similar to those of adults, and although Davis and Ruggiero (1973) have suggested that slower response speeds might contribute to deficits in the delayed-response performances of aged monkeys, it seemed unlikely that the present acquisition deficits were attributable to motor impairments. Because the concurrent procedures of this study were self-paced, both aged and adult subjects were permitted as much time as necessary to select an object, and all animals readily grasped and displaced the discriminanda appearing on the WGTA tray. Accordingly, motor-performance requirements involving skilled or rapid movement seemed minimal in the present concurrent tasks. Additionally, if aged animals had fatigued more rapidly or were less motivated than adults, then they might have been expected to perform especially poorly during the second daily repetition of the set of problems. However, aged subjects showed no less intrasession improvement than adults, and, thus, acquisition impairment did not appear to depend on an age-related characteristic assessed by this measure.

It has also been suggested that aged animals might show poorer discrimination based, not upon memory loss per se, but upon a decreased capacity or willingness to attend to tasks. One observation in support of this contention was that aged subjects performed relatively poorly on a delayed-response task when simply identifying a stimulus without an interposed delay (King \& Michels, 1989; Moss et al., 1988). As another example of attentional influences, Bartus and Dean (1979) reported that aged monkeys' delayed-response performances were impaired when distracting stimuli were presented during the delay interval, and they suggested that the impairment represented a heightened sensitivity to interference. In our concurrent testing situation, presenting several intervening problems between the subsequent reappearances of a problem might provide the kind of distraction capable of selectively impairing the attention of old animals. Indeed, distractions or presentation of competing stimuli may be one of the reasons why multiple-problem tasks, like concurrent discrimination, provide more sensitive detection of age effects than do single-problem tasks like Davis' (1974) pattern-generation problems or the pattern and object reversals used by Rapp (1990). However, with regard to the present results, it seems unlikely that a distraction or impaired-attention interpretation would account for the distinctive pattern of error shown by our old animals. Although one might speculate that disruption could selectively influence within- or between-session performances, it is difficult to comprehend how this could be restricted to one problem type (i.e., baited problems).

On the present tests of retention, the long-term memory that supported concurrent discrimination was not compromised by the aging process, and this outcome may help to clarify an earlier noted contrast of results. Recall that Medin et al. (1973) examined aged and adult monkeys' performances on concurrent tasks and concluded that aged animals were impaired by an intersession (24-h) retention loss, whereas Davis (1974, pp. 170-173) found that aging did not affect 24-h retention if both groups were equated on original learning. In view of these contrasts, differences between the retention-test interpretations of Medin et al. and the present study may depend exclusively on methodological issues. For Medin et al., aged monkeys' day-to-day improvement was compromised and termed a retention deficit, whereas we found that aged subjects required more errors to learn these tasks and viewed this as impaired acquisition. Perhaps both investigations reveal deficits in the aged monkey's ability to perform on concurrent tasks, and the differences in interpretation between these two studies hinge upon the procedural properties that define their acquisition or retention measures. Such a view provides some resolution of apparent differences in outcomes when age effects on memory for object discriminations are under evaluation.

The general issue of contrasts between acquisition and retention measures has also been noted when evaluating the effects of object preferences on concurrent performance. Bessemer and Stollnitz (1971) contended that object preferences were stern determinants of monkeys' concurrent retention performances, whereas Treichler et al. (1977) suggested that preference effects did not influence retention after extensive training. The issue appeared to be resolved by noting that, although object preferences were potent determinants of performance during the course of acquisition, these preferences did not yield enhanced error rates on retention tests administered after attainment of stern acquisition criteria (Treichler, Petros, \& Lesner, 1981).

In the present study, expression of object preferences seems to have made some contribution to the retentiontest results. With the imposition of a 2-week interval after attainment of a rather lenient acquisition criterion, the present retention test appeared to entail some relearning, as attested to both by the functions shown in Figure 1 and by the presence of significant improvement over the course of this test. The characterization of retention performance as relearning was also enhanced by the presence of a significant preference (baited/unbaited) effect during the retention test. The pattern of differences between last acquisition day scores and first retention day scores also lends credence to the view that retention performances entail substantial relearning.

As was done with the acquisition scores, we computed the average change in error between successive problem presentations during the retention test and analyzed these scores in an ANOVA. Comparisons of the various age groups on their within- versus between-sessions and baited versus unbaited conditions yielded no significant main effects or interactions, in part because of the overall low level of error. However, even here, the only combination of conditions that did not show a net reduction in error between successive presentations was the one where old animals performed between-session transitions on 
baited problems. Thus, although not of sufficient magnitude to yield significant differences, the pattern of interpresentation transitions was like that of acquisition.

Although we have suggested that age differences are more likely to be revealed within tests of acquisition, we must note that there were no age effects associated with the reacquisition performances seen in any of our retention tests. Despite the presence of slightly more error by aged animals on the first retention session, the two groups did not show significant differences, either on that day or on the reattainment of terminal criteria. Perhaps a retention test that required a greater relearning component might reveal age differences, but further empirical tests would be required to devise performance measures of sufficient sensitivity to detect such an effect.

When the full scope of the present acquisition and retention outcomes is viewed in the broader context of investigations of age effects in primates, it is striking to note the parallels between these results and those from a relatively recent report by King and Michels (1989). In both investigations, age influences on the nature of errors obtained in nonhuman primates' two-choice discrimination performance were under evaluation, and the two investigations reached some rather similar conclusions. Among these were the indication that greater error by older animals might not be attributable to a memory deficit, that older animals exhibit no selective susceptibility to a systematic perseverative error, and that under appropriate test conditions, aged animals commit more random errors than do younger monkeys. The last point is especially germane to the present study's finding that a major factor in characterizing old monkeys' performances was their lower probability of maintaining a systematic object preference between daily acquisition sessions; that is, on initial daily problem presentations, they tended toward randomness. Accordingly, both King and Michels and we emphasize the nature of systematic and nonsystematic performance error, not as "noise" in the system, but as an indicant of distinctive information-processing characteristics.

In overview, it seems clear that appropriately selected forms of the concurrent paradigm can provide effective behavioral assays for detecting age effects on nonhuman primate performance. On a hopeful note, this study suggests that some forms of concurrent retention are not compromised by the aging process, and if appropriate measures of performance transition are provided, concurrent discrimination by nonhuman primates may offer a comparative model for study of the aging process (OscarBerman, 1984).

\section{REFERENCES}

Bartus, R. T. (1979). Effects of aging on visual memory, sensory processing and discrimination learning in a nonhuman primate. In J. M. Ordy \& K. R. Brizzee (Eds.), Aging: Vol. 10. Sensory systems and communication in the elderly (pp. 85-114). New York: Raven.

Bartus, R. T. (1988). The need for common perspectives in the development and use of animal models for age-related cognitive and neurodegenerative disorders. Neurobiology of Aging, 9, 445-451.

Bartus, R. T., Dean, R. L. (1979). Recent memory in aged nonhuman primates: Hypersensitivity to visual interference during retention. Experimental Aging Research, 5, 385-400.

Bartus, R. T., Fleming, D., \& Johnson, H. R. (1978). Aging in the rhesus monkey: Debilitating effects on short-term memory. Joumal of Gerontology, 33, 858-871.

Bessemer, D. W., \& Stollnitz, F. (1971). Retention of discriminations and an analysis of learning set. In A. M. Schrier \& F. Stollnitz (Eds.), Behavior of nonhuman primates (Vol. 4., pp. 1-58). New York: Academic Press.

DAvis, R. T. (1974). Monkeys as perceivers. In L. A. Rosenblum (Ed.), Primate behavior: Vol. 3. Developments in field and laboratory research (pp. 1-263). New York: Academic Press.

Davis, R. T. (1978). Old monkey behavior. Experimental Gerontology, 13, 237-250.

Davis, R. T., \& Ruggiero, F. T. (1973). Memory in monkeys as a function of preparatory interval and pattern complexity of matrix displays. American Journal of Physical Anthropology, 38, 573-577.

KING, J. E., MicheLS, R. R. (1989). Error analysis of delayed response in aged squirrel monkeys. Animal Learning \& Behavior, 17, $157-162$.

Kubanis, P., \& Zornetzer, S. F. (1981). Age-related behavioral and neurobiological changes: A review with an emphasis on memory. Behavioral \& Neural Biology, 31, 115-172.

Medin, D. L. (1969). Form perception and pattern reproduction by monkeys. Journal of Comparative \& Physiological Psychology, 68, 412-419.

Medin, D. L., O’Neil, P., Smeltz, E., \& Davis, R. T. (1973). Age differences in retention of concurrent discrimination problems by monkeys. Journal of Gerontology, 28, 63-67.

Moss, M. B., Rosene, D. L., \& Peters, A. (1988). Effects of aging on visual recognition memory in the rhesus monkey. Neurobiology of Aging, 9, 495- 502 .

OSCAR-BERMAN, M. (1984). Comparative neuropsychology and alcoholic Korsakoff disease. In L. R. Squire \& N. Butters (Eds.), Neuropsychology of memory (pp. 194-202). New York: Guilford.

RAPP, P. R. (1990). Visual discrimination and reversal learning in the aged monkey (Macaca mulatta). Behavioral Neuroscience, 104, 876-884.

STEIN, D. G. (Ed.). (1980). The psychobiology of aging: Problems and perspectives. New York: Elsevier North-Holland.

TREICHLER, F. R. (1984). Long-term retention of concurrent discriminations by monkeys. Physiological Psychology, 12, 92-96.

Treichler, F. R., Petros, T. V., And Lesner, S. A. (1981). Preference effects on acquisition and retention of concurrent discriminations by rhesus monkeys. Animal Learning \& Behavior, 9, 401-405.

Treichler, F. R., Wetsel, W. C., \& Lesner, S. A. (1977). Some characteristics of concurrent discrimination and retention by monkeys. Learning \& Motivation, 8, 213-228.

(Manuscript received August 12, 1991; revision accepted for publication May 14, 1992.) 